\title{
Scanning deflectometric form measurement avoiding path-dependent angle measurement errors
}

\author{
Michael Schulz \\ Michael.schulz@ptb.de
}

\section{Gerd Ehret}

\section{Arne Fitzenreiter}

\author{
Physikalisch-Technische Bundesanstalt, Braunschweig, Germany
}

Physikalisch-Technische Bundesanstalt, Braunschweig, Germany

Physikalisch-Technische Bundesanstalt, Braunschweig, Germany

For the highly accurate topography measurement of nearly flat optical surfaces, scanning deflectometric methods are capable of achieving nanometer accuracy. In these systems, an autocollimator is typically used as the deflectometric sensor and a pentaprism is applied for the scanning process. When ultimate accuracy is desired, a drawback of these systems is that the autocollimator output signal often depends slightly on the optical path length, resulting in topography errors during scanning. Here, we present a new deflectometric method which separates the angle measurement from the scanning process and, thereby, avoids possible errors due to different optical path lengths. In contrast to conventional deflectometry, the new technique achieves an almost exact autocollimation by appropriately tilting the specimen during scanning. The tilt angle necessary to achieve autocollimation complies with the deflectometric angle determined in conventional deflectometry. The tilt angle is measured with an additional autocollimator at a fixed distance without errors due to different optical path lengths. The separation of angle measurement and the scanning process enable both tasks to be optimized independently. This opens up new possibilities of reducing lateral resolution by facilitating smaller apertures and of assessing topographies with larger curvatures. The concept was tested successfully by a demonstrator setup. The first measurements on a test specimen agree with results obtained with the established Extended Shear Angle Difference (ESAD) technique at the one nanometer level. [DOI: 10.2971/je0S.2010.10026]

Keywords: flatness measurement, deflectometry, topography

\section{INTRODUCTION}

In optical form metrology, a number of deflectometric techniques have been developed to measure the topography of nearly flat surfaces with uncertainties in the nanometer or even sub-nanometer range. Examples are the ESAD technique [1]-[7], the Nanometer Optical Component Measuring Machine (NOM) [8]-[11], and the Long Trace Profiler (LTP) $[12,13]$. These methods are applied for flatness calibrations and surface characterizations, for example, of synchrotron mirrors, optical elements for astronomy or interferometric flatness references. These techniques make use of the straight propagation of a light beam, emitted e.g. from an autocollimator, to establish a straightness reference. A pentaprism deflects the light at a right angle and directs it to the surface under test (SUT) (see Figure 1). The SUT reflects the beam back to the autocollimator (or another angle measuring device) to measure the angle of the local surface slope. The SUT is scanned by moving the pentaprism along the direction of the autocollimator's light beam. Hence, these conventional systems combine straightness representation and angle measurement in one instrument. In the following, autocollimators will be addressed, but the approach also applies to other kinds of optical angle measuring devices, e.g. LTPs. A drawback of angle measuring instruments used in this kind of application, in particular when nanometer accuracy is desired, is that the measurement results depend on the optical path length $[14,15]$ which inevitably changes during the scan-

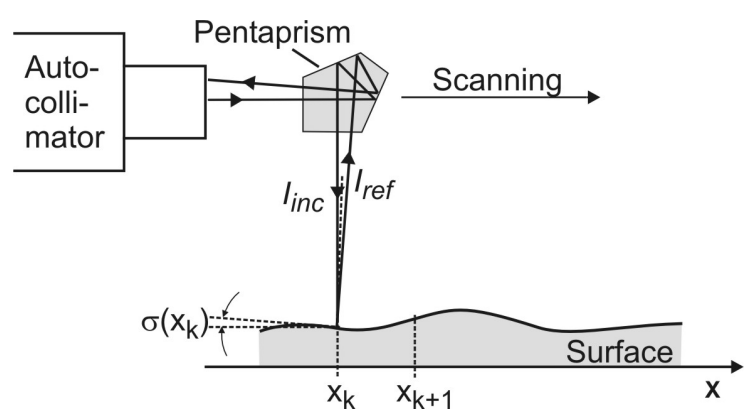

FIG. 1 Principle of conventional deflectometric procedures.

ning process. This effect leads to systematic deviations of the measured deflection angles and to corresponding topography errors. These deviations especially appear when non-zero deflection angles are measured. In these cases, the reflected beam passes the optical components (e.g. the autocollimator objective) at paths which differ from the optical axis and which depend on the position of the scanning pentaprism. Hence, any aberration or imperfection of the optical components will affect the back-propagating beam. A correction of this effect would require much effort to accurately calibrate the autocollimator for each possible distance. In this paper, a solution is described which decouples the scanning process from the an- 


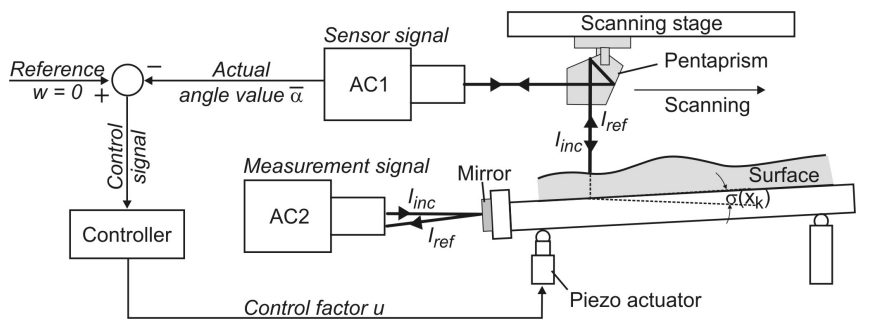

FIG. 2 Principle of operation of the EADS system. $A C_{1}$ : Straightness representation and null instrument, $\mathrm{AC2}$ : angle measurement.

gle measurement and, thus, avoids the necessity of distancedependent calibration of the angle measuring device.

\section{PRINCIPLE OF THE NEW DEFLECTOMETRIC SCANNING METHOD}

The basic idea of the new deflectometric scanning method is to separate the tasks of angle measurement and straightness representation. Figure 2 shows the principle of the setup. Here, the autocollimator AC1 (or a different optical angle measuring system) represents the straightness reference. Its light is directed to the SUT by the pentaprism. In contrast to conventional deflectometric concepts, the SUT is tilted by a tilting stage, for example by piezoelectric actuators. Any deviation from normal incidence is detected by AC1 and a feedback loop is used to tilt the SUT to achieve normal incidence and to maintain this condition (the application of such feedback loops is a well-known measurement tool, for example in Atomic Force Microscopy). Since AC1 is operated at zero deflection angle ('null detection'), the light always passes through AC1 and the pentaprism on the same optical path for all scanning positions. Thus, path-length-dependent errors are significantly reduced. The tilt angle of the SUT is measured subsequently by a second autocollimator AC2 at a fixed distance. The autocollimator AC2 can be operated in a mode that assures the highest accuracy, e.g. by using an optimized aperture and distance. For each scanning position, the feedback loop needs to work sufficiently accurately.

In this paper, this new deflectometric scanning method is named Exact Autocollimation Deflectometric Scanning (EADS), since an "exact" autocollimation, i.e. the autocollimator's object point is imaged into itself, is fundamental to this technique.

\section{DEMONSTRATOR EXPERIMENT OF THE EADS METHOD}

To test the new EADS method, a demonstrator experiment was set up and the first measurements were performed. For this purpose, the hardware of our ESAD setup [1] was modified (see Figure 3): the autocollimator of the ESAD setup was applied as a null detector (AC1) and the ESAD shearing stage was used as the linear scanning stage for the pentaprism. As this stage has a travel range of only $100 \mathrm{~mm}$, the specimen size was limited, but was nevertheless sufficient to demonstrate

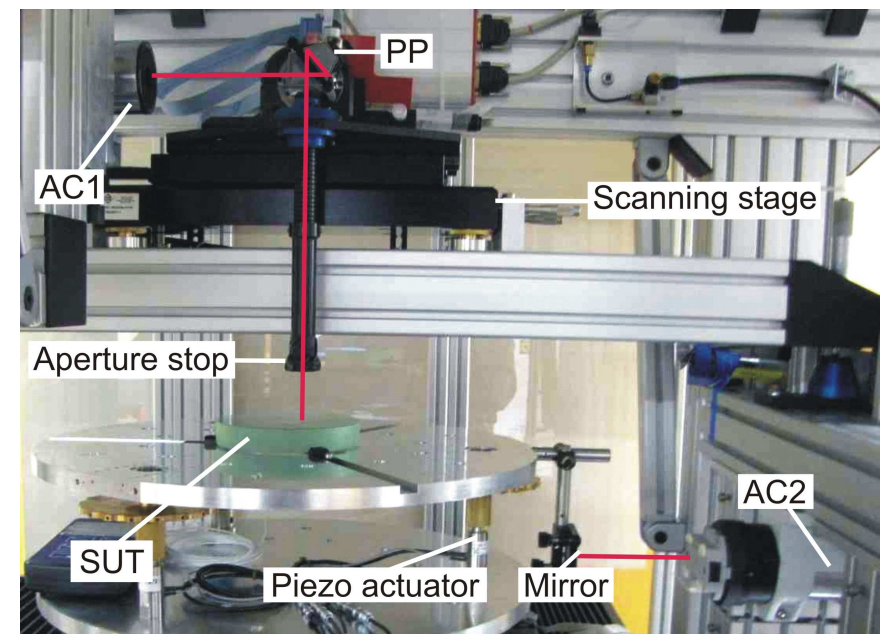

FIG. 3 Photo of the provisional setup to demonstrate the new EADS principle: Autocollimator as the null instrument ( $A C_{1}$ ), pentaprism (PP), linear scanning stage, mirror connected to the surface under test (SUT), tilt angle measuring autocollimator (AC2) and piezoelectric actuator for tilting. The beams of $A C_{1}$ and $A C_{2}$ are added schematically as red lines.

the measurement principle. A second autocollimator (AC2) was mounted to the frame of the setup to measure the tilt angle of the specimen. The mirror required was fixed to the specimen holder. Tilting the specimen was realized by the application of a piezoelectric actuator underneath the sample.

The SUT is tilted by the actuator unit, consisting of a piezoelectric actuator with control electronics. AC1 and AC2 are highly accurate autocollimators which have been calibrated by the manufacturer. The devices provide 25 readings per second and have been operated with an aperture of $5 \mathrm{~mm}$. For $\mathrm{AC} 1$, an aperture stop ( $5 \mathrm{~mm}$ diameter) was used near to the surface under test.

For the first demonstrations, the feedback loop (see Figure 2) was realized by a software controller. It is designed as a pure integral controller. The control signal is derived by the average $\bar{\alpha}$ of 25 angle readings from AC1. During the first control loop at time $t_{1}$, the controller uses the value $\bar{\alpha}\left(t_{1}\right)$ and calculates the output signal $u\left(t_{1}\right)=K_{1} \bar{\alpha}\left(t_{1}\right)$ used to drive the actuator unit. $K_{1}$ is the amplification factor of the integral controller and was determined experimentally. During the second loop, the autocollimator values at the times $t_{1}$ and $t_{2}$ are added (integral controller) resulting in a new output signal $u\left(t_{2}\right)=K_{1}\left[\bar{\alpha}\left(t_{1}\right)+\bar{\alpha}\left(t_{2}\right)\right]$. The control loop is repeated until the angle $\bar{\alpha}$ is approximately zero. The output signal for the actuator unit is computed as follows,

$$
u\left(t_{n}\right)=K_{1} \sum_{i=1}^{n} \bar{\alpha}\left(t_{i}\right)
$$

where $\bar{\alpha}\left(t_{i}\right)$ is the mean value of 25 angle values at time $t_{i}$, $u\left(t_{n}\right)$ is the output signal for the actuator unit at time $t_{n}$, and $K_{1}$ is the amplification factor of the integral controller.

After a few cycles, the control deviation is approximately zero $(\bar{\alpha}<0.01$ arcseconds). For the experiments we used seven cycles. Figure 4 shows, as an example, the results of a typical control process with seven cycles. 

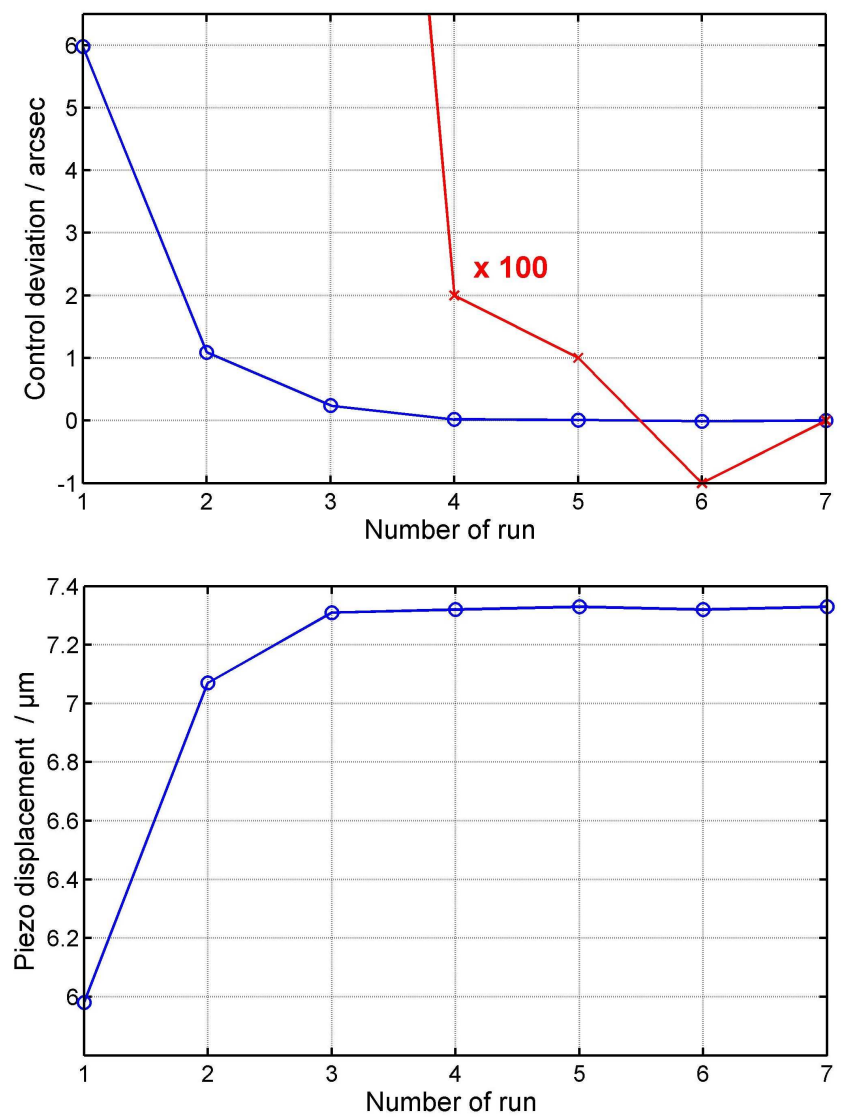

FIC. 4 Reading of $A C_{1}$ in a typical cycle of the control loop (top): The red curve shows the control deviations magnified by a factor of 100 . The corresponding displacement of the piezoelectric actuator is shown additionally (bottom). For better visibility, the data points at the seven positions are connected by solid lines.

The first topography measurements with the EADS setup were carried out using a quartz plate. Since the range of the applied piezo actuator was limited to about $15 \mu \mathrm{m}$, only a part of the specimen with a topography range of about $250 \mathrm{~nm}$ could be assessed. Figure 5 shows the results of this measurement. The left figure presents the averaged topography over seven single scans. The deviations of the individual scans to this average scan are shown in the right-hand part of Figure 5. These first examples demonstrate a repeatability of less than $1 \mathrm{~nm}$. Further improvements can be expected from optimizing the mechanical setup, especially the mirror mounting, the controller, etc.

The measured data agree within 0.04 arcseconds (deflection angles) and $1 \mathrm{~nm}$ (topography) with corresponding ESAD measurement results. The residual differences between both measurements are shown in Figure 6. This makes it clear that the new method yields measurement results that agree well with conventional ESAD.

\section{ADVANTAGES OF THE EADS PRINCIPLE}

In Section 3, the consistency of measurement results, in a case where both measurement principles can work, is presented. Beyond this, there are several advantages, which characterize the new EADS principle.
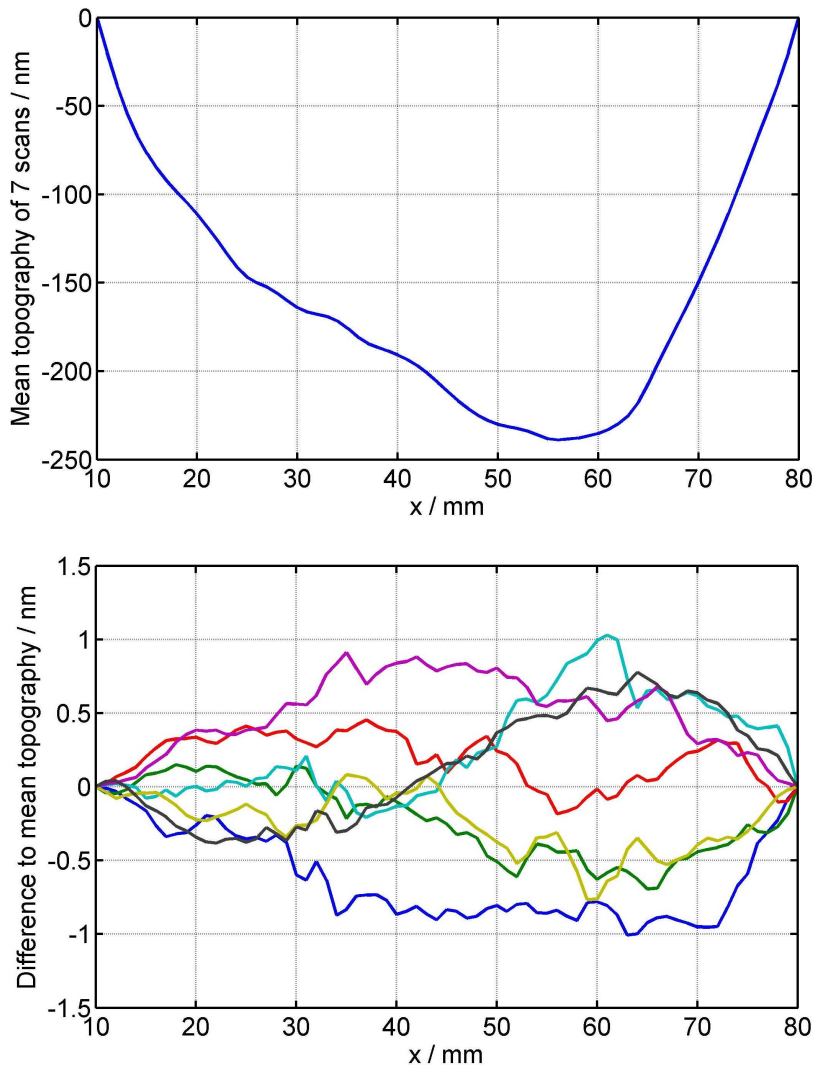

FIG. 5 Average of seven topography measurements with the EADS setup (top) and deviations of the single measurements from the average (bottom).
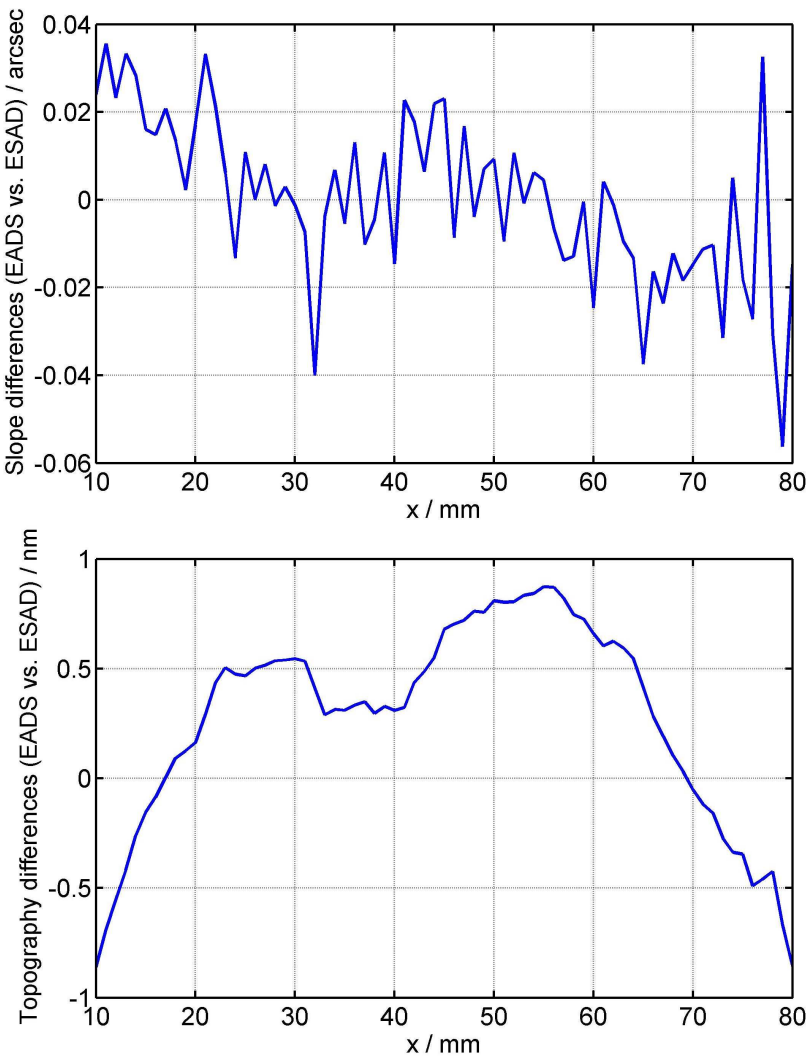

FIG. 6 Slope (top) and topography difference (bottom) between the EADS and ESAD measurements.

Since the new EADS principle allows one to separate the tasks of angle measurement and straightness representation, the 
new technique enables the optimization of both tasks. It is thus possible to eliminate distance-dependent effects of the angle measurement, which do not influence the measurement of the surface slope any more. Furthermore, it offers the possibility of enhancing the lateral resolution by using smaller apertures for the null detector which represents straightness. Devices other than autocollimators may also be applied as a null detector, for example long trace profilers or devices measuring laser beam displacements.

Since the surface under test is tilted so that the incoming beam is reflected back in exact autocollimation, any local deformation of the surface will result only in a symmetrical deformation of the reflected beam which should have only little influence on the null detector. Therefore, also topographies with larger curvatures (that could not be assessed with established deflectometric techniques due to the measurement range of the autocollimators) are assessable. Using a rotary stage with an angle encoder for turning the specimen and measuring the rotation angle could even extend the range of measurable surface forms. However, for large rotation angles, tilt and rotation are no longer equivalent and corresponding care has to be taken.

\section{CONCLUSIONS}

The principle of the new Exact Autocollimation Deflectometric Scanning has been presented and successfully tested in a demonstrator setup. The first comparison measurements on a test surface with the established deflectometric ESAD method showed a very good agreement within $1 \mathrm{~nm}$.

The EADS principle is a promising new technique offering novel possibilities and improved accuracies in optical form measurement. Compared to established deflectometric techniques, for example the ESAD technique, more strongly curved surfaces can be measured, the spatial resolution can be improved and optical errors of the system can be reduced. The EADS principle will also be implemented in the new deflectometric flatness reference at PTB [5]-[7].

\section{References}

[1] I. Weingärtner, and M. Schulz, "Novel scanning technique for ultraprecise measurement of slope and topography of flats, spheres and aspheres and complex surfaces" Proc. SPIE 3739, 274-282 (1999).
[2] I. Weingärtner, M. Schulz, and C. Elster, "Novel scanning technique for ultra-precise measurement of topography" Proc. SPIE 3782, 306-317 (1999).

[3] C. Elster, and I. Weingärtner, "Solution to the shearing problem" Appl. Opt. 38, 5024-5031 (1999).

[4] R. Geckeler, and I. Weingärtner, "Sub-nm topography measurement by deflectometry: Flatness standard and wafer nanotopography" Proc. SPIE 4779, 1-12 (2002).

[5] G. Ehret, M. Schulz, M. Stavridis, and C. Elster, "A new flatness reference measurement system based on deflectometry and difference deflectometry" in Fringe 2009: 6th International Workshop on Advanced Optical Metrology, W. Osten, M. Kujawinska, eds., 318-323 (Springer-Verlag, Berlin, 2009).

[6] G. Ehret, M. Schulz, M. Baier, and A. Fitzenreiter, "A new optical flatness reference measurement system" Proc. DGa0 110, P22 (2009).

[7] M. Schulz, G. Ehret, M. Stavridis, and C. Elster, "Concept, design and capability analysis of the new deflectometric flatness reference at PTB" Nucl. Instrum. Meth. A 616, 134-139 (2010).

[8] F. Siewert, T. Noll, T. Schlegel, T. Zeschke, and H. Lammert, “The nanometer optical component measuring machine: a new sub-nm topography measuring device for x-ray optics at BESSY" Proc. AIP 705, 847-850 (2004).

[9] H. Lammert, T. Noll, T. Schlegel, F. Siewert, and T. Zeschke, “Optisches Messverfahren und Präzisionsmessmaschine zur Ermittlung von Idealformabweichungen technisch polierter Oberflächen" Patent No. DE 10303659 (2005).

[10] F. Siewert, H. Lammert, and T. Zeschke, "The nanometer optical component measuring machine" in Modern Developments in XRay and Neutron Optics, A. Erko, M. Idir, T. Krist, A. G. Michette, eds., 193-200 (Springer-Verlag, Berlin, 2008).

[11] S. G. Alcock, K. J. S. Sawhney, S. Scott, U. Pedersen, R. Walton, F. Siewert, T. Zeschke, F. Senf, T. Noll, and H. Lammert, "The Diamond-NOM: A non-contact profiler capable of characterizing optical figure error with sub-nanometre repeatability" Nucl. Instrum. Meth. A 616, 224-228 (2009).

[12] P. Z. Takacs, S. Qian, and J. Colbert, “Design of a long-trace surface profiler" Proc. SPIE 749, 59-64 (1987).

[13] S. Qian, P. Z. Takacs, and W. Jark, "The penta-prism LTP: A long-trace-profiler with stationary optical head and moving penta prisma" Rev. Sci. Instrum. 66, 2562-2569 (1995).

[14] R. D. Geckeler, and A. Just, "Distance-dependent influences on angle metrology with autocollimators in deflectometry" Proc. SPIE 7077, 70770B (2008).

[15] F. Siewert, J. Buchheim, and T. Zeschke, "Characterization and calibration of 2nd generation slope measuring profiler" Nucl. Instrum. Meth. A 616, 119-127 (2010). 